\title{
UIR BANDS IN CARBON STAR SPECTRA.
}

\author{
A.K. SPECK \& M.J. BARLOW \\ Physics \& Astronomy Department \\ University College London \\ Gower Street \\ London \\ WC1E $6 B T$
}

\begin{abstract}
Unidentified infrared emission bands (UIR bands) have been attributed to polycyclic aromatic hydrocarbons (PAHs), which are believed to require ultraviolet radiation in order for the UIR bands to be excited. If, in addition to amorphous carbon and hydrogenated amorphous carbon (HAC) particles, PAHs are able to form in the outflows of cool carbonrich stars (Cherchneff et al. 1991), then the weak UV radiation field from such stars would be unlikely to be able to excite the UIR bands and so the PAH species could remain undetected in the spectra of C-stars. However, cool carbon stars with hot companions might be exposed to strong enough UV radiation fields for UIR-band emission to be excited from PAHs. Buss et al. (1991) reported the detection of the $8 \mu \mathrm{m}$ UIR-band (C-C stretch) in the IRAS LRS spectrum of HD 38218 (TU Tau), a carbon star with a hotter A2III companion. To investigate the phenomenon further, we have therefore obtained UKIRT CGS3 $10 \mu \mathrm{m}$ spectra of three carbon stars with hot companions, TU Tau, UV Aur and CS776. It was found that TU Tau showed the $11.25 \mu \mathrm{m}$ and $8.6 \mu \mathrm{m}$ UIR-bands (both attributed to C-H bend modes) at good contrast, while UV Aur clearly exhibited the $11.25 \mu \mathrm{m}$ UIR band. No narrow UIR-band emission was detected in the spectrum of CS776. We have fitted these $10 \mu \mathrm{m}$ region spectra using a $\chi^{2}$-minimization program equipped to fit stellar and dust emission continua together with the broad SiC feature and the narrow UIR-bands. The features seen in the spectra of TU Tau and UV Aur can be well fitted by a narrow $11.25 \mu \mathrm{m}$ UIR-band sitting on top of a broad, self-absorbed $11.3 \mu \mathrm{m}$ silicon carbide feature. Our results therefore provide strong support for the supposition that PAHs can form in carbon star outflows.
\end{abstract}




\section{Introduction}

The unidentified infrared bands (UIR bands) were first observed in the early 1970's in the spectra of planetary nebulae (Gillett et al. 1973; Merrill et al. 1975; Cohen et al. 1975; Russell et al. 1977a \& b). These bands are a family of narrow features, the major bands of which appear at 3.3, $3.4,6.2,7.7,8.6$ and $11.3 \mu \mathrm{m}$, although there are many minor bands and broad plateaux. Since their discovery, they have been observed in the spectra of many other objects (e.g. post-AGB objects, reflection nebulae, HII regions and active galaxies). They have long been attributed to polycyclic aromatic hydrocarbons or PAHs in regions which have strong UV fields (Léger \& Puget 1984; Duley \& Williams 1981). The mechanism by which the UIR bands are believed to be produced involves a PAH absorbing a UV photon, becoming excited and redistributing the energy to its vibrational modes, then re-emitting in the infrared. The exact structure and origin of these PAH species is not known, although it has been suggested that they form in the outflows of carbon-rich AGB stars (Jura 1987). Because of the mechanism by which the UIR bands are produced, we do not expect to see them in the spectra of carbon stars as these objects have insufficient UV radiation. However, carbon stars in binary systems with hot companions could exhibit UIR bands, as these carbon stars are exposed to a source of UV radiation. There have been various models proposed for the formation of PAHs in the outflows of carbon stars. Both Keller (1987) and Gail and Sedlmayr (1987) used classical nucleation theory to model the formation of PAHs and concluded that PAH formation is possible in the outflows of carbon stars. Both Frenklach \& Feigelson (1989) and Cherchneff et al. (1991) investigated PAH production in these regions using a kinetic approach rather than relying on classical nucleation theory. Broadly their findings agreed although Cherchneff et al. (1991) suggested that these models may be missing certain mechanisms which may favour PAH growth. Cadwell et al. (1994) discussed induced nucleation of PAHs in carbon rich circumstellar envelopes, where the grains are formed by deposition onto a "seed". Their model suggested that PAHs could form a mantle around a seed grain. They then suggested two scenarios for the production of UIR band carriers. Firstly, the aromatic molecules attached to the grains that are ejected into the ISM may be desorbed by various mechanisms, and then the free PAHs give rise to the UIR bands; second, the aromatics near the surface of the depositional grains give rise to the UIR bands without desorption. The latter scenario fits the HAC hypothesis of Duley \& Williams (1981). 


\section{Target Selection}

Buss et al. (1991) examined the IRAS spectra of carbon stars in binary systems with hot companions, with a view to observing UIR bands. All the stars chosen by Buss et al. have A star companions. One star in their sample, TU Tau, overlaps with the present sample. Another star that Buss et al. considered but did not include was UV Aur, dismissed as being a symbiotic star. However, we believe that this classification is incorrect and that UV Aur is, in fact, a visual binary with a main sequence B star companion. It is therefore included in the present sample. The third star in our sample is CS 776, a binary with an A star companion. This source was observed by Le Bertre (1990), who detected anomalously strong diffuse interstellar bands (DIBs) in the spectrum of pf the A star. It has been suggested (Léger \& d'Hendecourt 1985; van der Zwet \& Allamandola 1985) that the likely carriers of the DIBs are PAHs. If the DIBs are in fact due to PAHs forming around the carbon star, then it is not unreasonable to assume that there could also be UIR bands evident in the infrared spectrum of CS 776 .

\section{The Investigation}

We have executed an 8-13 $\mu \mathrm{m}$ survey of 31 carbon stars with a view to investigating the silicon carbide $(\mathrm{SiC})$ features in their spectra (Speck et al. 1996; Speck et al. 1997, hereafter SBS97). Amongst these stars we found 4 which exhibited $\mathrm{SiC}$ in net absorption, one that was distinctly unusual, while the remaining 26 showed $\mathrm{SiC}$ emission features. Amongst these $\mathrm{SiC}$ emission sources were the three stars with hot companions, TU Tau, UV Aur and CS 776 These three stars have been investigated in a variety of ways. Firstly, all three of the spectra exhibit a broad silicon carbide feature at about $11.3 \mu \mathrm{m}$. In order to discover whether there is also a narrow $11.3 \mu \mathrm{m}$ feature that can be associated with the UIR band, the profile of the $\mathrm{SiC}$ feature must be well characterised. To this end, each of the three spectra were compared to the spectra of the 23 other carbon stars with $\mathrm{SiC}$ emission features discussed by SBS97, as well as to each other. These comparisons showed that both TU Tau and UV Aur exhibit an additional narrow feature at about $11.3 \mu \mathrm{m}$ over and above the $\mathrm{SiC}$ feature while CS 776 shows no extra features so that the broad $11.3 \mu \mathrm{m}$ feature in its spectrum can be attributed to $\mathrm{SiC}$ alone. In addition, TU Tau may also exhibit a feature at $8.6 \mu \mathrm{m}$. The work of SBS97 included the $\chi^{2}$-fitting of $\mathrm{SiC}$ features in carbon stars. They fitted both TU Tau and UV Aur using a spectrum which was edited (i.e. the section of spectrum between 11.0 and $11.5 \mu \mathrm{m}$ was removed), so that the suspected UIR band did not affect the fitting of the SiC feature. Thus, the fits to TU Tau and UV Aur were for the $\mathrm{SiC}$ feature only and did not include any part of the UIR band. We 

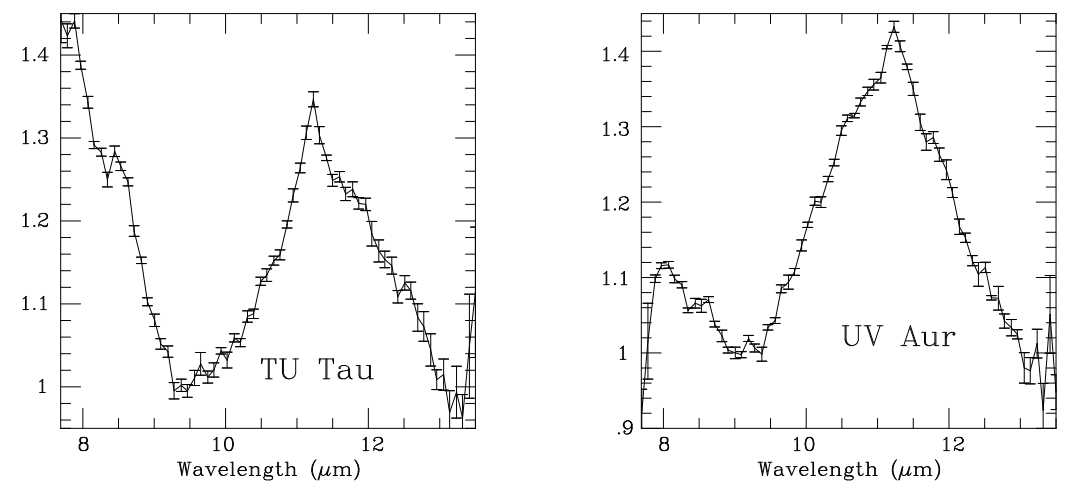

Figure 1. Normalised spectra of TU Tau and UV Aur

have used these fits to isolate the UIR bands, by subtracting the fit to the $\mathrm{SiC}$ feature from the original flux-calibrated spectrum of each source. The resultant isolated narrow features can be seen in Fig. 1.

The normalised spectrum of TU Tau shows a clear feature at $11.13 \mu \mathrm{m}$, together with a steep rise in the continuum shortward of $9 \mu \mathrm{m}$ and a likely $8.6 \mu \mathrm{m}$ feature. The "normalised" spectrum of UV Aur is slightly more unexpected. This also shows a clear feature at $11.13 \mu \mathrm{m}$, however there are equally strong features evident at about 10.04 and $10.49 \mu \mathrm{m}$. There is no rise in the continuum shortward of $9 \mu \mathrm{m}$ and no sign of a feature at $8.6 \mu \mathrm{m}$. We then proceeded to fit the $11.3 \mu \mathrm{m}$ UIR band in these spectra using the $\chi^{2}$-fitting program described in SBS97 which was equipped with the ability to fit a narrow UIR feature at $11.25 \mu \mathrm{m}$. The first attempt at fitting was performed using the flux-calibrated spectrum over the entire wavelength range, 7.5-13.5 $\mu \mathrm{m}$, using a blackbody and an $\mathrm{SiC}$ emission feature, as described by SBS97, together with the $11.25 \mu \mathrm{m}$ UIR band. This fitting resulted in no solution for TU Tau. UV Aur was fitted and the results are presented in Table 1. We then proceeded to attempt the fitting again, this time restricting the fit to the 9.5 to $13.5 \mu \mathrm{m}$ region. The reason for this restriction of the wavelength range is to minimize the effects of spurious features between 7.5 and $9.5 \mu \mathrm{m}$ with which the fitting program is not equipped to deal (see SBS97). This proved more successful for TU Tau but this time it was UV Aur that was unable to be fitted. Again, the results of these fits are presented in Table 1. Finally, following the success of SBS97 in fitting the $\mathrm{SiC}$ features using self-absorbed silicon carbide, the fitting of the UIR band was attempted using a blackbody, self-absorbed $\mathrm{SiC}$ emission and the UIR band. This produced by far the best results for both sources, which are plotted in Fig. 2. The results can be seen in the last two rows of Table 1. We also attempted to fit TU Tau using a $8.6 \mu \mathrm{m}$ UIR feature, but 
TABLE 1. Results of the $\chi^{2}$-fitting of the flux-calibrated spectra

\begin{tabular}{|c|c|c|c|c|c|c|c|}
\hline Source & $T_{\text {colour }}^{\dagger}$ & Wavelength range $(\mu \mathrm{m})$ & SiC type & $T_{B B}(\mathrm{~K})$ & $T_{S i C}(K)$ & $\tau_{S i C}$ & $\chi_{R}^{2}$ * \\
\hline UV Aur & 1500 & $7.5-13.5$ & $\beta-\mathrm{SiC}^{2}$ & 1469 & 217 & & 3.218 \\
\hline TU Tau & 1100 & $7.5-13.5$ & - & - & - & & - \\
\hline UV Aur & 1500 & $9.5-13.5$ & - & -- & - & & - \\
\hline TU Tau & 1100 & $9.5-13.5$ & $\beta-\mathrm{SiC}$ & 936 & 184 & & 2.958 \\
\hline UV Aur ${ }^{1}$ & 1500 & $9.5-13.5$ & $\mathrm{SiC}-600^{2}$ & 5189 & 989 & 0.194 & 0.366 \\
\hline $\mathrm{TU} \mathrm{Tau}^{1}$ & 1100 & $9.5-13.5$ & Pég-SiC ${ }^{3}$ & 2311 & 440 & 0.538 & 0.333 \\
\hline
\end{tabular}

TABLE 1. Results of the $\chi^{2}$-fitting of the flux-calibrated spectra

$\dagger$ The colour temperature is taken from SBS97

$\star \chi_{R}^{2}$ is the reduced $\chi^{2}$ value, i.e. $\chi^{2} / n$ where $n$ represents the number of degrees of freedom.

1 These fits use self-absorbed $\mathrm{SiC}$

2 ' $\beta$-SiC' \& 'SiC-600' are from Borghesi et al.(1985)

3 'Pég-SiC' is the feature from Pégourié (1987)
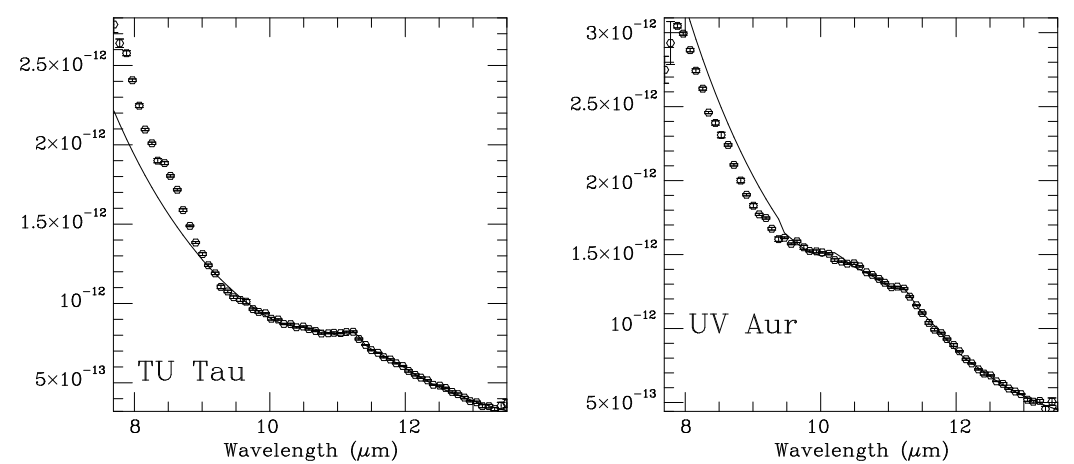

Figure 2. $\chi^{2}$-fits to the UIR band in the spectra of TU Tau and UV Aur

this was unsuccessful for both $\mathrm{SiC}$ in pure emission and self-absorption.

\section{Discussion}

As can be seen from the fits to UU Aur and TU Tau in Fig. 2, we have found convincing evidence for the presence of the $11.3 \mu \mathrm{m}$ UIR band in the spectra of two carbon stars with A- or B-type companions as sources of UV radiation. The lack of a feature in the spectrum of CS 776 is surprising, however the detection of DIBs in the spectrum of its companion star may be important. It is also surprising that we cannot fit a $8.6 \mu \mathrm{m}$ feature to the two spectra which exhibit the $11.3 \mu \mathrm{m}$ UIR band as these are usually expected together. There is a likely $8.6 \mu \mathrm{m}$ feature in the spectrum of TU Tau, and our inability to fit it may be model based. There is no sign of such a feature, however, in the spectrum of UV Aur. This could be a contrast problem, or 
it could be due to the nature of the PAHs responsible for each band. The 8.6 and $11.3 \mu \mathrm{m}$ bands are both attributed to aromatic C-H modes. However it has been suggested that the $8.6 \mu \mathrm{m}$ band is due to ionised PAHs, whereas the $11.3 \mu \mathrm{m}$ band is due to neutral PAHs (Szczepanski \& Vala 1993). If the $8.6 \mu \mathrm{m}$ feature is present in the spectrum of TU Tau, this implies that our three carbon stars with hot companions may have different states of ionization, possibly due to differences in the companion star. It may be that UV Aur is the least ionized and therefore only shows the $11.3 \mu \mathrm{m}$ bands, while TU Tau is more ionised, which is why it exhibits a weak $8.6 \mu \mathrm{m}$ band.

\section{References}

Borghesi, A., Bussoletti, E., Colangeli, L., De Blasi, C. (1985) Astron. Astrophys., 153, 1 Bregman, J.D., Allamandola, L.J., Tielens, A.G.G.M., Geballe, T.R., Witteborn, F.C. (1989) Astrophys. J., 344, 791

Buss, R.H., Tielens, A.G.G.M., Snow, T.P. (1991) Astrophys. J., 372, 281

Cadwell, B.J., Wang, H., Feigelson, E.D., Frenklach, M. (1994) Astrophys. J., 429, 285

Cherchneff, I., Barker, J.R., Tielens, A.G.G.M. (1992) Astrophys. J., 401, 269

Cohen, M., Fawley, W.M., Anderson, C.M., Cowley, A., Coyne, G.V. Gull, T.R., Harlan, E.A., Herbig, G.H., Holden, F., Hudson, H.S. (1975) Astrophys. J., 196, 179.

Cohen, M., Tielens, A.G.G.M., Allamandola, L.J. (1985) Astrophys. J., 299, L93

Duley, W.W., Williams, D.A. (1981) Mon. Not. R. Astron. Soc., 196, 269

Frenklach, M., Feigelson, E.D. (1989) Astrophys. J., 341, 372

Gail, H.P., Sedlmayr, E. (1985) Astron. Astrophys., 148, 183

Gillett, F.C., Forrest, W.J., Merrill, K.M. (1973) Astrophys. J., 183, 87

Jura, M. (1987) in Polycyclic Aromatic Hydrocarbons and Astrophysics, eds. A. Léger, L. d'Hendecourt and N. Boccara, Reidel, Dordrecht, p. 3

Keller, R. (1987) in Polycyclic Aromatic Hydrocarbons and Astrophysics, eds. A. Léger, L. d'Hendecourt and N. Boccara, Reidel, Dordrecht p. 387

Le Bertre, T. (1990) Astron. Astrophys., 236, 472

Léger, A., Puget, J.L. (1984) Astron. Astrophys., 137, L5

Merrill, K.M., Soifer, B.T., Russell R.W. (1975) Astrophys. J., 200, L37

Pégourié, B. (1988) Astron. Astrophys., 194, 335

Russell, R.W., Soifer, B.T., Merrill, K.M. (1977) Astrophys. J., 213, 66

Russell, R.W., Soifer, B.T., Willner, S.P. (1977) Astrophys. J., 217, L149

Speck, A.K., Barlow, M.J., Skinner, C.J. (1996) in From Stardust to Planetessimals: Contributed Papers, eds. M.E. Kress, A.G.G.M. Tielens and Y.J. Pendleton, (NASA CP-3343) p. 61

Speck, A.K., Barlow, M.J., Skinner, C.J. (1997) Mon. Not. R. Astron. Soc., in press.

Szczepanski, J., Vala, M. (1993) Astrophys. J., 414, 646

van der Zwet, G.P., Allamandola, L.J. (1985) Astron. Astrophys., 146, 76. 